\title{
ORIGINAL
}

\section{CONOCIMIENTO DE LA ENFERMEDAD DE CHAGAS POR PARTE DE LOS PROFESIONALES SANITARIOS DE TRES HOSPITALES EN LA PROVINCIA DE ALMERÍA (*)}

\author{
Ma José Muñoz-Vilches (1), Joaquín Salas-Coronas (2), María Isabel Gutiérrez-Izquierdo (3), David \\ Metz (4), Jorge Salvador-Sánchez (5) y Francisco Giménez-Sánchez (6).
}

(1) Área Integrada de Pediatría. Unidad de Medicina Tropical. Hospital de Poniente. El Ejido, Almería.

(2) Unidad de Medicina Tropical. Hospital de Poniente. El Ejido, Almería.

(3) Facultad de Ciencias de la Educación, Enfermería y Fisioterapia. Departamento de Enfermería y Fisioterapia. Universidad de Almería.

(4) Área Integrada de Ginecología. Hospital de Poniente. El Ejido, Almería.

(5) Servicio de Pediatría. Hospital La Inmaculada. Huercal-Overa, Almería.

(6) Servicio de Pediatría. Hospital Torrecárdenas. Almería.

(*) El trabajo fue financiado por el Proyecto Salud e Inmigración (Ref 2010-2-13 y Ref 2011-2-1) subvencionado por el Centro de Estudios de Migraciones y Relaciones Interculturales (CEMyRI), en colaboración con la Universidad de Almería, el Ministerio de Trabajo e Inmigración y el grupo de investigación CTS-582.

\section{RESUMEN}

Fundamentos: Los profesionales sanitarios de la provincia de Almería atienden a pacientes con enfermedades importadas para las que en muchas ocasiones carecen de suficiente formación. El objetivo del estudio fue valorar los conocimientos que tienen sobre diferentes aspectos de la enfermedad de Chagas los profesionales médicos y de enfermería que trabajan en tres hospitales de Almería atendiendo a mujeres gestantes.

Método: estudio descriptivo trasversal realizado en el año 2011 a través de un cuestionario, anónimo y voluntario, a 278 sanitarios (personal médico y personal de enfermería) del área materno-infantil de los tres hospitales de la provincia. En el hospital de Poniente se instauró en 2007 un programa de despistaje de la enfermedad en mujeres embarazadas. Para el análisis estadístico, las variables cuantitativas se describieron con la media y su desviación estándar. Para la comparación de las variables cualitativas se utilizó la prueba $\mathrm{chi}^{2}$ de Pearson o la prueba exacta de Fisher según correspondiera.

Resultados: $116(41,7 \%)$ profesionales aceptaron participar en el estudio. $80(69 \%)$ fueron mujeres y $36(31 \%)$ hombres, con edad media de 36,78 años. El personal médico presentó una media de respuestas correctas del $73,9 \%$ y el personal de enfermería del $50,7 \%$. El hospital de Poniente tuvo mayor porcentaje de respuestas correctas en aspectos relativos a la distribución geográfica de la enfermedad $(73,7 \%)$, los mecanismos de transmisión (86\%) y el diagnóstico $(82,5 \%)$.

Conclusiones: Los profesionales del hospital de Poniente presentan de forma general un mejor conocimiento de la enfermedad de Chagas en comparación con los profesionales de los otros dos centros hospitalarios, lo que probablemente se relaciona con la existencia del programa de despistaje de la enfermedad.

Palabras clave: Enfermedad de Chagas. Trasmisión vertical. Trypanosoma cruzy. Inmigrantes. Inmigración. Mujeres embarazadas.

\section{Correspondencia}

Joaquín Salas Coronas

C/ Los Califas $n^{\circ} 8$

04720 Aguadulce

Almería

\section{ABSTRACT}

\section{Health Professionals' Knowledge on} Chagas Disease in the Province of Almeria, Spain

Background: Health professionals who care for patients with imported diseases often lack enough training. The aim of the study is to assess the knowledge of Chagas disease among doctors and nurses attending at-risk pregnant women in our province.

Method: descriptive study through a performed anonymous and voluntary knowledge questionnaire for 278 physicians and nurses working at maternity and children's health services in the three hospitals in the province. In Poniente Hospital was established in 2007 a program of screening for the disease in pregnant women. For statistical analysis, quantitative variables were described using the mean and standard deviation. For comparison of qualitative variables we used the chi-square test or Fisher exact test as appropriate. Differences in age and years of experience depending on the hospital were measured by Brown-Forsythe robust test.

Results: $116(41.7 \%)$ professionals agreed to participate in the study. 80 $(69 \%)$ were women and $36(31 \%)$ men, mean age 36.78 years. By professional categories, physicians have a mean of $73.9 \%$ correct responses, the nurses $50.7 \%$. Poniente Hospital had the highest percentage of correct answers on aspects of the geographical distribution of the disease $(73.7 \%)$, the mechanisms of transmission ( $86 \%$ ) and diagnosis $(82.5 \%)$.

Conclusions: The Poniente Hospital professionals generally have a better Knowledge about Chagas disease compared with two other professionals hospitals, which probably is related to the existence of the screening program for the disease.

Keyword:Chagas disease. Vertical Infection Transmission. Trypanosoma cruzi. Immigrants. Immigration. Pregnant women. 


\section{INTRODUCCIÓN}

La Tripanosomiasis Americana o enfermedad de Chagas es una zoonosis endémica de los países continentales de Centroamérica y Sudamérica, donde se considera un problema importante de salud públi$\mathrm{ca}^{1,2}$. Debido al fenómeno migratorio, la enfermedad de Chagas ha comenzado a ser un problema de salud pública también en países no endémicos ${ }^{3-5}$. En estos últimos, donde no existe el vector transmisor de la enfermedad, la transmisión puede producirse mediante la transfusión de hemoderivados, el trasplante de órganos, accidentes de laboratorio y de madre a hijos durante la gestación ${ }^{3,6,7}$.

En España se están desarrollando en los últimos años diversas acciones para evitar la transmisión de la enfermedad por las distintas vías $^{8-11}$. Sin embargo, en lo referente a la transmisión vertical, sólo se ha implementado un programa de control a nivel institucional en dos Comunidades Autónomas ${ }^{10-11}$. En España, la transmisión vertical es de especial importancia al ser el principal país europeo receptor de inmigración procedente de Latinoamérica ${ }^{12-13}$, existiendo actualmente más de 2 millones de personas nacidas en sus países, de las que el $55 \%$ son mujeres. De hecho, cada vez es mayor el número de casos adquiridos por esta vía descritos en la literatura ${ }^{14-17}$.

De manera pionera en Andalucía, el hospital de Poniente de Almería comenzó a realizar el despistaje de la enfermedad de Chagas en mujeres embarazadas latinoamericanas de modo protocolizado en abril del año $2007^{18}$. Sin embargo, en el resto de centros hospitalarios de la provincia, y en gran parte de España, no se ha implantado ningún protocolo de estas características, por lo que el cribado de la enfermedad depende en gran medida del interés y el conocimiento de la enfermedad por parte de los profesionales.

El objetivo del estudio fue evaluar los conocimientos que tienen sobre diferentes aspectos de la enfermedad de Chagas los profesionales sanitarios que trabajan en tres hospitales deAlmería atendiendo a mujeres gestantes.

\section{SUJETOS Y MÉTODOS}

La población objeto de estudio fueron los profesionales sanitarios (médicos, matronas, enfermeras y auxiliares) implicados en los cuidados materno-infantiles que desempeñan su labor asistencial en los tres hospitales públicos de la provincia de Almería: Hospital de Poniente (El Ejido), Hospital Torrecárdenas (Almería) y Hospital de La Inmaculada (Huercal-Overa).

Se llevó a cabo un estudio descriptivo transversal a través de un cuestionario desarrollado por los investigadores del proyecto, cuya cumplimentación fue de carácter voluntario y anónimo (anexo 1). Dicho cuestionario evaluó los conocimientos de los profesionales sobre diferentes aspectos de la enfermedad de Chagas, como la epidemiología (preguntas 1 , 2 y 3 ), clínica y diagnóstico (preguntas 4 , 5 y 6) y tratamiento de la enfermedad (preguntas 7 y 8). El cuestionario recogió además datos relacionados con la categoría profesional, entre los que se incluyeron los años de labor asistencial en el puesto de trabajo y la formación académica.

Para facilitar el análisis de los resultados sobre los conocimientos de la enfermedad de Chagas se dividieron en cuatro bloques: resultados globales, por hospitales, por categorías profesionales y por especialidad médica.

El estudio se llevó a cabo en el año 2011, durante 2 semanas consecutivas, entre los profesionales de cada uno de los tres hospitales en las Áreas de Toco-Ginecología y Pediatría (incluyendo planta de hospitalización, paritorios y consultas externas). Se consideró este período el adecuado para poder incorporar el mayor 
número posible de profesionales, dado el carácter de trabajo rotatorio de turnos que tienen gran parte de estos trabajadores. Los criterios de selección de la muestra fueron desarrollar su trabajo en el área materno-infantil de los centros seleccionados, pertenecer a las categorías profesionales objeto del estudio (médicos, enfermeros y matrones) y la voluntariedad para completar el cuestionario. Su correcta cumplimentación fue supervisada por investigadores del proyecto que entregaban los cuestionarios y esperaban su finalización para recogerlos en ese mismo momento.

Para el análisis estadístico se utilizó el programa SPSS para Windows en su versión 17.0 Las variables cuantitativas se describieron usando la media y su desviación estándar. Para las variables cualitativas se obtuvieron las frecuencias absolutas y relativas expresadas en porcentajes. Para la comparación de las diferencias entre las variables cualitativas se utilizó la prueba $\mathrm{chi}^{2}$ de Pearson o la prueba exacta de Fisher según correspondiera. Para las comparaciones dos a dos en la tabla 3 se ha utilizado la prueba de comparación de proporciones $\mathrm{Z}$, usando en este caso el nivel de significación de $p<0,016$. Las diferencias de edad y años de experiencia en fun- ción del hospital de origen se midieron mediante la prueba robusta de BrownForsythe. Para todas las pruebas realizadas se consideró un nivel de significación estadística para las diferencias de $\mathrm{p} \leq 0,05$.

El estudio fue aprobado por la Comisión de Ética e Investigación del Hospital de Poniente.

\section{RESULTADOS}

De los 278 profesionales incluidos en el estudio (89 médicos, 142 enfermeros y 47 matronas), 116 (41,7\%) cumplieron finalmente los criterios de inclusión con la siguiente tasa de participación por centros: Hospital de Poniente 57 (89\%), Hospital Torrecárdenas 24 (14\%) y Hospital La Inmaculada 35 (79,5\%).

Las categorías profesionales a las que pertenecían las personas encuestadas fueron $47(40,5 \%)$ médicos/as (29 especialistas en Pediatría y 18 especialistas en Ginecología y Obstetricia) y $69(59,5 \%)$ enfermeras/os (39 trabajadores de las áreas de paritorio, hospitalización Ginecológica y hospitalización de Pediatría y 30 de la especialidad de matrona). La distribución de dichas categorías por hospitales se muestra en la tabla 1. Todo el personal médico excepto dos ginecólogos accedie-

Tabla 1

Distribución de los participantes por hospitales, categoría profesional y especialidad

\begin{tabular}{|l|c|c|c|c|}
\hline & $\begin{array}{c}\text { Hospital } \\
\text { de Poniente }\end{array}$ & $\begin{array}{c}\text { Hospital } \\
\text { la Inmaculada }\end{array}$ & $\begin{array}{c}\text { Hospital } \\
\text { Torrecárdenas }\end{array}$ & Total \\
\hline $\mathrm{N}^{\text {o de profesionales }}$ & $57(49,1 \%)$ & $35(30,2 \%)$ & $24(20,7 \%)$ & 116 \\
\hline Médicos (total) & $24(20,7 \%)$ & $11(9,5 \%)$ & $12(10,3 \%)$ & $47(40,5 \%)$ \\
\hline -Pediatras & $7(6 \%)$ & $5(4,3 \%)$ & $6(5,2 \%)$ & $18(15,5 \%)$ \\
\hline -Ginecólogos & $17(14,6 \%)$ & $6(5,2 \%)$ & $6(5,2 \%)$ & $29(25 \%)$ \\
\hline Enfermeros (total) & $33(28,5 \%)$ & $24(20,7 \%)$ & $12(10,3 \%)$ & $69(59,5 \%)$ \\
\hline Matronas & $13(11,2 \%)$ & $9(7,8 \%)$ & $8(6,9 \%)$ & $30(25,9 \%)$ \\
\hline Planta y paritorios & $20(17,2 \%)$ & $15(13 \%)$ & $4(3,4 \%)$ & $39(33,6 \%)$ \\
\hline Edad media en años (DE) & $34,72(6,992)$ & $37,32(6,786)$ & $41,09(11,662)$ & $36,78(8,376)$ \\
\hline Años experiencia profesional (DE) & $10,86(6,422)$ & $13,92(7,668)$ & $21,56(11,768)$ & $14,02(9,048)$ \\
\hline
\end{tabular}

(DE): desviación estándar 
ron a la especialidad vía de Médicos Internos Residentes (MIR).

Del total de profesionales $80(69 \%)$ fueron mujeres y $36(31 \%)$ hombres, con una edad media de 36,78 años. Se observaron diferencias estadísticamente significativas en la edad y la experiencia laboral de los profesionales del Hospital de Poniente frente a los profesionales del Hospital Torrecárdenas, siendo los primeros más jóvenes y con menos años de experiencia profesional (edad media: Hospital de Poniente 34,72 años; Hospital Torrecárdenas 41,09 años, $\mathrm{p}<0,002$. Experiencia profesional: Hospital de Poniente: 10,86 años; Hospital Torrecárdenas 21,6 años, $\mathbf{p}<0,001)$. Los profesionales del Hospital La Inmaculada también presentaban menos años de experiencia laboral en comparación con los del H. Torrecárdenas (H. la Inmaculada 13,92 años; H. Torrecárdenas 21,56 años; $\mathrm{p}<0,003$ ). El tipo de contrato más frecuente en todas las categorías fue el laboral fijo (60,3\%).

No se encontraron diferencias estadísticamente significativas al analizar las respuestas al cuestionario en función de la edad y el sexo de los profesionales.

Los resultados globales, que incluyen los tres hospitales y todas las categorías profesionales, se muestran en la tabla 2 en la que se puede observar:
1.- El porcentaje de respuestas correctas de los profesionales en lo referente a los mecanismos de transmisión y el diagnóstico (preguntas 2,3 y 5) fue del 78,4\%, 71,6\% y $70,7 \%$ respectivamente.

2.- El porcentaje de profesionales que conocía las manifestaciones clínicas fue del 54,3\%.

3.- En relación con el tratamiento de la enfermedad, el porcentaje de respuestas correctas fue del 41,4\%. En este apartado se hizo referencia a la efectividad y capacidad curativa del tratamiento en fases agudas (se incluyó la transmisión vertical).

4.- El 50\% de los profesionales conocía que el tratamiento es más efectivo y mejor tolerado en los niños que en los adultos.

Los resultados obtenidos en cada hospital se muestran en la tabla 3. En 7 de las 8 preguntas realizadas, el porcentaje de respuestas correctas fue superior entre los profesionales del Hospital de Poniente respecto a los otros dos centros. Las diferencias fueron estadísticamente significativas en las cuestiones relacionadas con la distribución de la enfermedad (Hospital de Poniente 73,7\%; Hospital La Inmaculada 48,6\%; Hospital Torrecárdenas 29,2\%, $\mathrm{p}=0,001$ para la diferencia del H.de

Tabla 2

Resultados de las respuestas del conjunto de los profesionales encuestados

\begin{tabular}{|l|c|c|}
\hline & $\begin{array}{c}\text { Respuestas correctas } \\
(\mathrm{n}=116)\end{array}$ & $\begin{array}{c}\% \\
\text { respuestas correctas }\end{array}$ \\
\hline Pregunta 1. La enfermedad de Chagas se distribuye por: & 66 & 56,9 \\
\hline Pregunta 2. El mecanismo de transmisión en países endémicos es: & 91 & 78,4 \\
\hline Pregunta 3. Se puede transmitir mediante: & 83 & 71,6 \\
\hline Pregunta 4. Respecto a las manifestaciones clínicas: & 63 & 54,3 \\
\hline Pregunta 5. El diagnóstico se realiza mediante: & 82 & 70,7 \\
\hline Pregunta 6.Una persona con enfermedad de Chagas: & 67 & 57,8 \\
\hline Pregunta 7. Respecto al tratamiento: & 48 & 41,4 \\
\hline Pregunta 8. La enfermedad de Chagas en los niños: & 58 & 50 \\
\hline
\end{tabular}


Tabla 3

Resultados de las encuestas en función del centro hospitalario

\begin{tabular}{|c|c|c|c|c|c|c|c|}
\hline & \multicolumn{2}{|c|}{$\begin{array}{c}\text { Hospital de } \\
\text { Poniente } \\
(\mathrm{n}=57)\end{array}$} & \multicolumn{2}{|c|}{$\begin{array}{c}\text { Hospital } \\
\text { La Inmaculada } \\
(\mathrm{n}=35) \\
\end{array}$} & \multicolumn{2}{|c|}{$\begin{array}{c}\text { Hospital } \\
\text { Torrecárdenas } \\
(\mathrm{n}=24) \\
\end{array}$} & \multirow{2}{*}{$\mathrm{p}$} \\
\hline & $\begin{array}{c}\text { respuestas } \\
\text { correctas }\end{array}$ & $\%$ & $\begin{array}{c}\text { respuestas } \\
\text { correctas }\end{array}$ & $\%$ & $\begin{array}{c}\text { respuestas } \\
\text { correctas }\end{array}$ & $\%$ & \\
\hline Pregunta 1. Enfermedad de Chagas se distribuye por: & 42 & 73,7 & 17 & 48,6 & 7 & 29,2 & $0,001 *$ \\
\hline Pregunta 2. Mecanismo de transmisión en países endémicos: & 47 & 82,5 & 28 & 80 & 16 & 66,7 & 0,278 \\
\hline Pregunta 3. Se puede transmitir mediante: & 49 & 86 & 21 & 60 & 13 & 54,2 & $0,003 *$ \\
\hline Pregunta 4. Respecto a las manifestaciones clínicas: & 35 & 61,4 & 17 & 48,6 & 11 & 45,8 & 0,314 \\
\hline Pregunta 5.El diagnóstico se realiza mediante: & 47 & 82,5 & 23 & 65,7 & 12 & 50 & $0,010 * *$ \\
\hline Pregunta 6.Una persona con enfermedad de Chagas: & 37 & 64,9 & 17 & 48,6 & 13 & 54,2 & 0,282 \\
\hline Pregunta 7.Respecto al tratamiento: & 27 & 47,4 & 11 & 31,4 & 10 & 41,7 & 0,321 \\
\hline Pregunta 8.La enfermedad de Chagas en los niños: & 29 & 50,9 & 16 & 45,7 & 13 & 54,2 & 0,802 \\
\hline
\end{tabular}

* Diferencias estadísticamente significativas del Hospital de Poniente respecto a los otros dos centros.

** Diferencias estadísticamente significativas entre Hospital de Poniente y Hospital Torrecárdenas.

Poniente respecto a los otros dos centros), el mecanismo de transmisión en zonas no endémicas [Hospital de Poniente 86\%; Hospital La Inmaculada $60 \%$; Hospital Torrecárdenas $54,2 \%$, $(\mathrm{p}=0,003$ significación estadística de la diferencia entre el $\mathrm{H}$ de Poniente y los otros dos centros)] y el método diagnóstico [Hospital de Poniente 82,5\%; Hospital La Inmaculada 65,7\%; Hospital Torrecárdenas $50 \%, \mathrm{p}=0,010$ significación estadísti- ca de la diferencia entre el $\mathrm{H}$ de Poniente y los otros dos centros)]. En este último caso, sólo se observaron diferencias estadísticamente significativas entre el Hospital de Poniente y el Hospital Torrecardenas.

Los resultados obtenidos en relación con la categoría profesionales se muestran en la tabla 4. Los médicos presentaron diferencias estadísticamente significativas

Tabla 4

\section{Resultados de las encuestas en función de la categoría profesional sin diferenciar por especialidad}

\begin{tabular}{|c|c|c|c|c|c|}
\hline & \multicolumn{2}{|c|}{$\begin{array}{c}\text { Personal médico } \\
\mathrm{n}=47\end{array}$} & \multicolumn{2}{|c|}{$\begin{array}{c}\text { Personal de enfermería } \\
n=69\end{array}$} & \multirow{2}{*}{$\mathrm{p}$} \\
\hline & $\begin{array}{c}\text { respuestas } \\
\text { correctas }\end{array}$ & $\%$ & $\begin{array}{c}\text { respuestas } \\
\text { correctas }\end{array}$ & $\%$ & \\
\hline Pregunta 1.La Enfermedad de Chagas se distribuye por: & 28 & 59,6 & 38 & 55,1 & 0,772 \\
\hline Pregunta 2. Mecanismo de transmisión en países endémicos: & 44 & 93,6 & 47 & 68,1 & 0,002 \\
\hline Pregunta 3. Se puede transmitir mediante: & 39 & 83,0 & 44 & 63,8 & 0,041 \\
\hline Pregunta 4. Respecto a las manifestaciones clínicas: & 35 & 74,5 & 28 & 40,6 & 0,001 \\
\hline Pregunta 5. El diagnóstico se realiza mediante: & 36 & 76,6 & 46 & 66,7 & 0,344 \\
\hline Pregunta 6. Una persona con Enfermedad de Chagas: & 40 & 85,1 & 27 & 39,1 & $<0,001$ \\
\hline Pregunta 7. Respecto al tratamiento: & 27 & 57,4 & 21 & 30,4 & 0,007 \\
\hline Pregunta 8. La enfermedad de Chagas en los niños. & 29 & 61,7 & 29 & 42 & 0,059 \\
\hline
\end{tabular}


en el porcentaje de respuestas correctas frente a los enfermeros en las preguntas relativas al mecanismo de transmisión en países endémicos (personal médico 93,6\%; personal de enfermería $68,1 \%, \mathrm{p}=0,002$ ); mecanismo generales de transmisión (médicos $83 \%$; enfermeros $63,8 \%, p=0,041)$; manifestaciones clínicas (médicos 74,5\%; enfermeros $40,6 \%, p=0,001)$; trasmisión de la enfermedad (médicos 85,1\%; enfermeros 39,1\%, $\mathrm{p}<0.001$ ) y tratamiento (médicos $57,4 \%$; enfermeros $30,4 \%, p=0,007)$.

El análisis de los datos por especialidad médica (Pediatría y Ginecología) no mostró diferencias estadísticamente significativas entre los profesionales de ambos grupos (datos no mostrados).

\section{DISCUSIÓN}

Los resultados de nuestro trabajo muestran que los profesionales que trabajan en un centro donde se ha implantado un programa de cribado de la enfermedad de Chagas tienen un mejor conocimiento de la enfermedad si los comparamos con aquellos que trabajan en hospitales donde este programa no existe. Este hecho es de especial interés al tratarse de una enfermedad que supone actualmente un importante problema de salud pública.

La evaluación sobre los conocimientos de la enfermedad de Chagas en profesionales de la salud que trabajan en áreas endémicas se realiza fundamentalmente para valorar la efectividad de las medidas de control vectorial implementadas en áreas específicas ${ }^{19-20}$. Sin embargo, apenas existe este tipo de estudios en zonas no endémicas. Dos trabajos en Estados Unidos evaluaron el conocimiento de los distintos especialistas que atienden a pacientes con riesgo de padecer enfermedad de Chagas. Ambos concluyen que existe un déficit generalizado en el conocimiento acerca de dicha enfermedad en la profesión médica, especialmente entre la comunidad de obstetras y ginecólogos ${ }^{21-22}$. Esta falta de conocimientos entre estos profesionales es de especial importancia, ya que la enferme- dad de Chagas se puede transmitir de madres a hijos.

En nuestro estudio los profesionales sanitarios encuestados presentan una edad media inferior a cuarenta años, pero con un tiempo medio de experiencia profesional superior a diez años. El gran flujo migratorio hacia España se inició en el año 1998. Por este motivo, los profesionales sanitarios deberían estar actualizados y adaptados a la realidad asistencial que la inmigración nos ofrece. Sin embargo, la formación específica en enfermedades importadas es en general muy deficitaria tanto durante el período de la licenciatura en las Facultades de Medicina o las Escuelas de Enfermería como en la formación postgrado. El hecho de que exista una Unidad de Medicina Tropical en el Hospital de Poniente que realiza actividades formativas dirigidas a sus profesionales, es probablemente el principal factor que contribuye a que el conocimiento de la enfermedad de Chagas en estos profesionales sea superior a los que prestan su asistencia en los otros dos centros hospitalarios. También influyen en este sentido el establecimiento de un programa de cribado de la enfermedad desde el 2007 y el hecho que el Hospital de Poniente atiende a una población inmigrante mayor que el resto de hospitales estudiados.

De forma global, los profesionales de todas las categorías tienen un mejor conocimiento sobre la distribución, transmisión y el diagnóstico de la enfermedad de Chagas que sobre otros aspectos de esta, como es el tratamiento. Estos resultados son probablemente el reflejo de la actividad que desarrollan estos profesionales en sus respectivos servicios en relación a la enfermedad de Chagas, que es el diagnóstico, pero no el seguimiento y tratamiento de los sujetos afectados.

Otro dato a destacar de nuestro estudio es que el personal médico conoce mejor la enfermedad de Chagas que los profesionales de enfermería y las matronas, especialmente en los aspectos de clínica y tratamiento. Esto por una parte es lógico ya que los diagnósti- 
cos clínicos y las prescripciones de tratamientos a pacientes la realizan el personal médico.

Si comparamos los resultados de nuestro trabajo sobre el conocimiento de la enfermedad en el personal médico, se puede interpretar que ginecólogos y pediatras de nuestro entorno muestran un mejor conocimiento de la enfermedad de forma global en comparación con trabajos anteriores realizados en EEUU, donde se constataba un déficit generalizado en el conocimiento acerca de la enfermedad de Chagas, especialmente entre los especialistas en ginecología ${ }^{21,22}$.

El dato más preocupante que podemos extraer de nuestro trabajo es que los profesionales, tanto médicos como enfermeros, que tratan directamente con mujeres gestantes en riesgo de padecer la enfermedad y transmitirla a sus hijos, tienen el mayor déficit de conocimientos en aspectos relacionados con el tratamiento y la repercusión de la enfermedad en los niños. El que casi el 50\% desconozca estos aspectos puede dar lugar a que niños que adquieren la enfermedad en nuestro medio y que en muchos casos van a vivir en nuestro país gran parte de su vida, no van a ser diagnosticados o no van a recibir un tratamiento adecuado que evite la aparición de graves complicaciones cardíacas o digestivas en el futuro.

Las limitaciones del estudio vienen dadas por una parte por el tamaño muestral, ya que resultó pequeño para poder obtener significación estadística en algunos de los resultados de nuestro trabajo. Este hecho es especialmente relevante entre los profesionales del Hospital Torrrecárdenas, con un índice de participación muy bajo. Como ya indicamos, la participación de los profesionales fue totalmente voluntaria. El hecho de que los profesionales que más participaron en el estudio fueran los del Hospital de Poniente es debido, probablemente, a estar más familiarizados con la enfermedad y, en general, con la atención a pacientes con enfermedades importadas, aunque también pueden haber sobrestimado los datos globales sobre el conocimiento de la enfermedad. El Hospital de La Inmaculada, segundo en índice de participación, atiende a una elevada proporción de población latinoamericana. En cualquier caso, pensamos que este trabajo es una primera aproximación al conocimiento que tienen nuestros profesionales sobre la enfermedad de Chagas, siendo necesario nuevos estudios que completen esta visión.

Como conclusión podemos decir que la deficitaria formación sobre la enfermedad de Chagas que tienen los profesionales sanitarios españoles que atienden a mujeres gestantes con riesgo de tener la enfermedad y a sus hijos hace necesaria la puesta en marcha de actividades formativas con objeto de ampliar dichos conocimientos, así como concienciar a las autoridades sanitarias de la necesidad de que los programas de control de la transmisión vertical de Chagas se extiendan por todo el territorio español. Será entonces cuando estemos cerca de evitar la transmisión en nuestro país de una enfermedad de fácil diagnóstico y curable en edades tempranas de la vida.

\section{AGRADECIMIENTOS}

Agradecemos a Pablo Garrido Fernández, técnico en metodología de la investigación y bioestadística de FIBAO, su apoyo inestimable en el tratamiento estadístico de los datos.

\section{BIBLIOGRAFÍA}

1. Organización Panamericana de la Salud. Misión Internacional de Evaluación de la situación epidemiológica y de control de la Enfermedad de Chagas en Bolivia. La Paz: OPS; 2011 [Citado el 20/05/2011].Disponible en: http://www.ops.org.bo/textocompleto/nchagas32348.pdf 
2. CLAP-OPS/OMS. Consulta Técnica sobre Información, Educación y Comunicación (IEC) en Enfermedad de Chagas Congénita. Rev Chil Infect. 2008; 25: 194-9.

3. Yadon ZE, Schmunis GA. Congenital Chagas disease: estimating the potential risk in the United States.Am J Trop Med Hyg. 2009; 81:927-33.

4. Bern C, Montgomery S, Herwaldt B, Rassi A Jr, Marin-Neto JA, Dantas RO et al. Evaluation and Treatment of Chagas Disease in the United States. A Systematic Review. JAMA. 2007; 298:2171-81.

5. Gascón, J. Diagnóstico y tratamiento de la Enfermedad de Chagas Importada. Documento de consenso. Med Clin (Barc). 2005; 125:130-5.

6. Piron M, Vergés M, Muñoz J, Casamitjana N, Sanz S, Maymó RM, et al. Seroprevalence of Trypanosoma cruzi infection in at-risk blood donors in Catalonia (Spain). Transfusion. 2008; 48:1862-8.

7. Muñoz J, Coll O, Juncosa T, Vergés M, del Pino M, Fumado V, et al. Prevalence and vertical transmission of Trypanosoma cruzi infection among pregnant Latin American women attending 2 maternity clinics in Barcelona, Spain. Clin Infect Dis. 2009; 48:173640.

8. Ministerio de Sanidad y Política Social. Enfermedad de Chagas y donación de Sangre. Informe Grupo de trabajo Donación de Sangre e Inmigración. Madrid: Ministerio de Sanidad y Política Social; 2009. Disponible en: http://www.msc.es/profesionales/saludPublica/medicinaTransfusional/publicaciones/docs/informeChagasJulio09.pdf

9. Boletín Oficial del Estado. Real Decreto $1088 / 2005$ del 16 de septiembre por el que se establecen los requisitos técnicos y condiciones mínimas de la hemodonación y de los centros y servicios de transfusión. Boletín Oficial del Estado núm 225 de 20/09/2005.

10. Direcció General de Planificació i Avaluació. Direcció General de Salut Pública. Departament de Salut. Protocol de Cribratge i Diagnóstic de Malaltia de Chagas en Dones Embarassades llatinoamericanes i en el sseus nadons. Barcelona: Departament de Salut; 2009.

11. Comunidad Valenciana. Generalitat. Protocolo de Chagas Importada. Valencia: Generalitat; 2009.

12. Instituto Nacional de Estadística. Padrón Municipal Madrid: INE; 2010. [citado el 12/06/2011]. Disponible en: http://www.ine.es
13. Gascón J, Pinazo MJ. Control de la transmisión vertical de Trypanosoma cruzi en España: principal reto de la patología importada. Enferm Infecc Microbiol Clin. 2008; 26:607-8.

14. Riera C, Guarro A, Kassab HE, Jorba JM, Castro M, Angrill R et al. Congenital transmission of Trypanosoma cruzi in Europe (Spain): a case report. Am J Trop Med Hyg. 2006;75: 1078-81.

15. Flores-Chávez M, Faez Y, Olalla JM, Cruz I, Gárate T, Rodríguez M et al. Fatal congenital Chagas disease in a non-endemic area: a case report. Cases J. 2008; $1: 302$.

16. Carrilero B, Quesada JJ, Alfayate S, Segovia M. Congenital Chagas disease in a newborn of a Bolivian mother. Enferm Infecc Microbiol Clin. 2009 ; 27:486-7.

17. Parada MC, Paredes C. Chagas vertical: una realidad en España Acta Pediatr Esp. 2007; 65: 565-9.

18. Muñoz-Vilches MJ, Salas J, Cabezas T, Metz D, Vázquez J, Soriano MJ. Chagas screening in pregnant Latin-American women. Experience in Poniente Almeriense (Almeria, Spain). Enferm Infecc Microbiol Clin. 2012; 30:380-2.

19. Villela MM, Pimenta DN, Lamounier PA, Dias JC. Avaliação de conhecimentos e práticas que adultos e criançastêm acerca da doença de Chagas e seus vetores em região endêmica de Minas Gerais, Brasil. Cad Saúde Pública. 2009; 25: 1701-10.

20. Villela MM, Souza JM, Melo Vde P, Dias JC. Chagas disease epidemiological surveillance in a decentralized program: evaluation of practice and knowledge among municipal health agents in an endemic region of Minas Gerais State, Brazil. Cad Saúde Pública. 2007; 23:2428-38.

21. Stimpert KK, Montgomery SP. Physician Awareness of Chagas Disease, USA. Emerg Infect Dis. 2010; 16: 871-872.

22. Verani JR, Montgomery SP, Schulkin J, Anderson B, Jones JL. Survey of Obstetrician-Gynecologists in the United States About Chagas Disease Am J Trop Med Hyg. 2010; 83:891-5. 


\section{Anexo 1}

\section{Cuestionario sobre conocimientos de la enfermedad de Chagas}

Seleccionar sólo una opción (la correcta está en negrita)

1.La enfermedad de Chagas se distribuye por:
a. Casi toda Latinoamérica
b. África subsahariana
c. África subsahariana y Latinoamérica
d. Todo el trópico
e. Toda América

2. El mecanismo principal de transmisión de la enfermedad de Chagas en los países endémicos es:
a. La vía sexual.
b. Ingesta de alimentos contaminados
c. Mordedura de un reptil
d. A través de un insecto vector.
e. A través de transfusiones sanguíneas.

3. La enfermedad de Chagas se puede transmitir mediante:
a. Transfusiones sanguíneas
b. Transmisión materno-fetal.
c. Ingesta de alimentos contaminados.
d. Accidentes de laboratorio
e. Todas las anteriores.

4. Con respecto a las manifestaciones clínicas de la enfermedad de Chagas.
a. Las más frecuentes son las cutáneas
b. Puede afectar a corazón, esófago e intestino
c. Generalmente se cura en la infancia
d. Produce clínica en todos los infectados
e. La mayoría fallece por problemas digestivos

5. El diagnóstico de la enfermedad de Chagas crónica se realiza mediante:
a. Aislamiento de los tripanosomas en heces
b. Biopsia cutánea
c. TAC abdominal con presencia de lesiones típicas
d. Serología sanguínea
e. Realización de cultivos

6. Una persona con enfermedad de Chagas:
a. Puede contagiar a su pareja
b. No puede donar sangre.
c. En caso de parto se recomienda cesárea electiva
d. En caso de embarazo siempre va a contagiar a su hijo
e. Tiene una esperanza de vida inferior a 10 años.

7. Con respecto al tratamiento de la enfermedad de Chagas:
a. No existe tratamiento
b. Es curativo en fases agudas
c. En la fase crónica nunca es eficaz.
d. Se requiere tratamiento de por vida.
e. El tratamiento en los adultos es eficaz en casi el 100\% de los casos

8. La enfermedad de Chagas en los niños:
a. Suele producir graves malformaciones.
b. Es imposible de diagnosticar.
c. No tiene tratamiento.
d. Es más fácil de tratar que en los adultos.
e. Los niños no padecen la enfermedad de Chagas 